\title{
The Introduction of building a Cylindrical Multi Display System by Game Engine
}

\author{
Hyungjin Jeon ${ }^{1}$, Eeljin $\mathrm{Chae}^{2}$, Hongsik $\mathrm{Pak}^{2}$ and Taesoo Yun ${ }^{2}$ \\ Division of Visual Contents, Graduate School, \\ Dongseo University, Busan, Korea

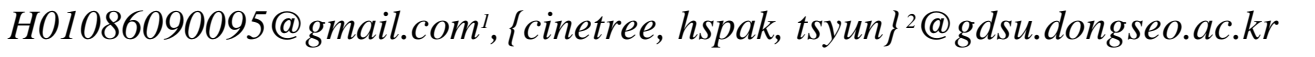 \\ Abstract
}

A new playground in which the interactive attributes of the game engine are applied has been created and its combination with a three-dimensional screen displov, which is realized through the state-of-the-art technology, has become widety, used. While the dome system has limits to extend its marketability due to its unnatural expression in three dimensions, there have been an increasing number of cases to install the system operated in the way to project through a cylindrical silver screen which makes it easier to realize the dimensional structures. The concept of a multi projector display and the principle of segmenting a 360-degree picture by dividing a frustum by angle, which is hoy the camera of a game engine is realized, are identical. In addition, the efficiency of the real-time rendering that utilizes a game engine with comparison to the rendering with the use of graphics software has been verified.

Keywords: Multi display, Game Engine, Interaction

\section{Introduction}

There has been an improvement in the exhibition method to effectively introduce history, sightseeing and cultaral materials-while 4D theaters and urban theme spaces have constantly been built accordingly. The three-dimensional screening system which is suitable for realistic visual experiences has wide been used in a three-dimensional experience theater with the basic projection system equipped since the 1980's. Since 'Avatar,' a sensational stereoscopic feature directed by James Cameron that broke the world's box office records, was released in 2009 and around that time, three-dimensional images became popular. This thesis offers ways for the stereoscopic image projection method on a cylindrical screen which uses the expression function of stereoscopic image and multi display solution to maximize its outstanding inturtive and visual-perceptual experiences compared to the flat display [1].

As-the $8 \mathrm{G}$ production-based software which has been constantly improved has widely been used in combination with the high-performance hardware in the display area, diverse and unque new media contents have naturally been used consequently in the operation system base. Such trend made it possible for contents production including games, animations, films and advertisements to make new ways to express images and improve the free visual concentration level by the use of contents which has caused the demand for experience-typed contents to increase. The multi display area, in particular, which was dominated by the images through pre-rendering process of image-based software, has come to a point where game solutions such as real-time hardware image rendering which utilizes the excellent image generation function of GPU play an important role [2].

Thus, this study introduces the partitioning of camera view of the screen in regards to the system design of cylindrical display such as $180 \sim 360$ degree and how to realize it. This 
system can allow to maximize the viewer's convenience at movements and visual sensation and immersion in theme parks' attraction.

This can also be referred as basic data for the establishment of display environment which is necessary to express high-quality experience-typed contents along with the innovation of multi display area that utilizes game engines. This thesis contains the following details; Chapter 2 introduces the methods of related studies; Chapter 3 describes the main technology used to realize the targeted system of '360 degree cylindrical display' and its design and the overall structure for implementation; Chapter 4 is about the rendering excellence and installation of multi display system; and Chapter 5 explains the conclusion of this study and further linked technological development of contents business models.

\section{Related Studies}

There has been a number of studies on the participatory exhibition space or the application of a mobile interface to the Kinect gesture interaction or to a large multi touch screen interaction, which makes it possible for an interaction to be properly realized for its particular purpose - a playground [3]. For example, there are ways to eontrol a display by location tracking with the use of a built-in camera or to recognize the actions of a user by an acceleration sensor or a user can utilize and interact with the UI which expresses its functions through buttons or dials for direct operation, in a space where devices are combined [4].

\subsection{Existing Technologies}

Since the early 2000's, studies on the distributed rendering to establish a system and segmenting super-resolution images have been given great attention [5]. Since the image expression is done by software rendering the high cost of building up rendering equipment during the production stage and the long processing time for high-resolution image data are an inevitable burden forproduction [6].

\subsection{Improved Technologies}

The technologies for improvement in this study utilize game engines. Generally the multi display is designed and setup in a way to project images through the camera's partition of 360-degree pictures with the subject as the center - and the projector and display panel which belong to each client connected to the server. Various combinations can be made depending on the image quality of project that uses the circle-vision through the guideline provision of picture production which is optimized for the circle-vision hardware and to the diameter of screen; each case includes different methods of producing image contents and rendering and also the cost of hardware and software that realize this accordingly.

Man specific technologies include the connection function for natural blending effects of the images projected on the projector linked to each client and some other functions for adjustment for correct four-side location of images that have been output. The utilization of game engines enables the major asset data such as modeling, texture, animation, etc., to freely modify and re-use as well as writing and data arrangement at the same time. It also allows the system to express real time IG (Image Generation) through the hardware rendering and to have many more advantages especially for the production of experience-typed interactive contents which analyze and apply the user's behavioral reactions to the contents.

Therefore, guidelines can also be offered so that anyone can produce optimized images of the standard of circle vision according to the technology to implement the cylindrical multi display system suggested in the study. 


\section{System Structure}

\subsection{Introduction to the System Configuration}

The diagram of cylindrical multi display system with high concentration level that this thesis suggests is shown in (Figure 1). When the cylindrical displayed view of $6.35 \mathrm{~m}$ in diameter is established, images are projected on each screen $(2.49 \mathrm{~m}$ in width) partitioned in eight areas of 19.939 in circumference at an angle of 45 degree(FOV) through eight projectors thus realizing the panorama-formed image view.

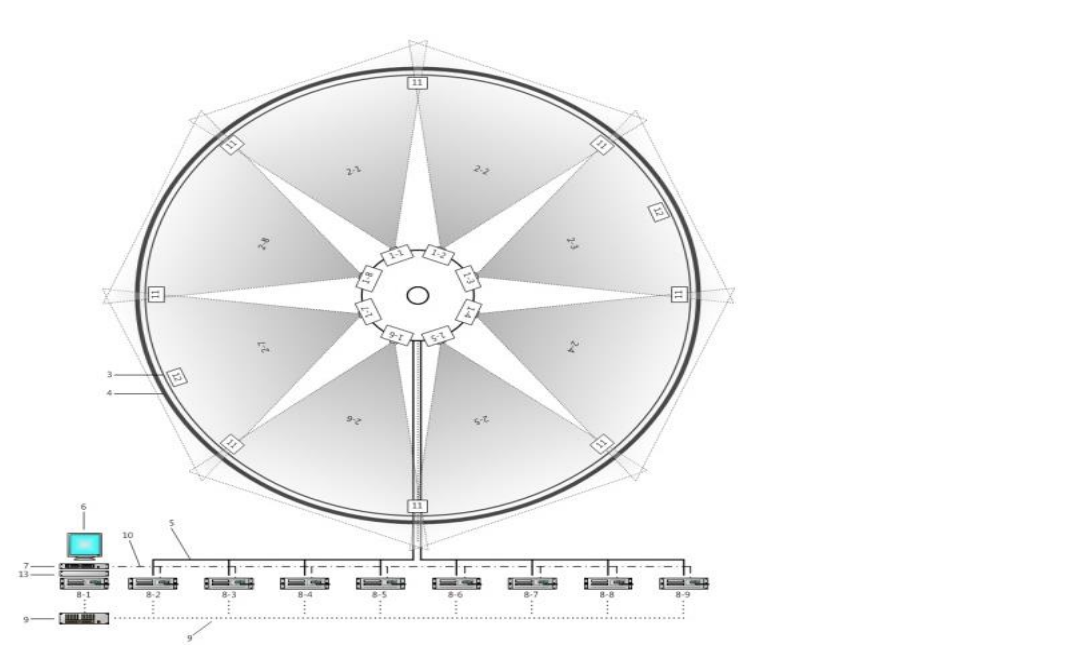

Figure 1. The Projector Structure of the Configuration System

\subsection{Image Configuration}

The determination of image sizes and proportions has a correlation which greatly affects the hardware election, image quality, etc. In this study, a seamless panoramic picture is printed visually on the cylmaridal screen by the ratio of 1:8 for the length and width of a basic image as shown below in Eigure 2 and the size of each image is the standard size designated in advance with the gameengine and projector lens [7].

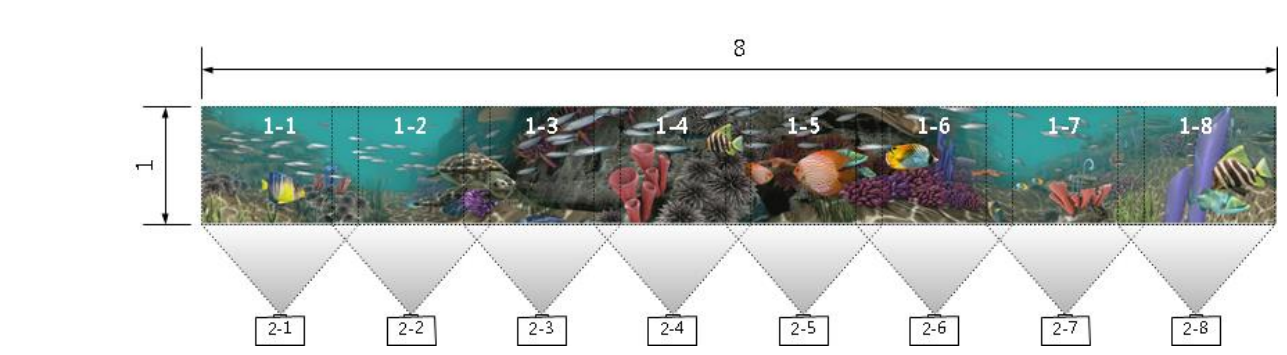

Figure 2. Ratio of Images

In accordance with Figure 2 illustrated above, the ratio of 1:8 is based on the proper quantity of projectors used for the 360-degree zone. Figure 3, the figure on the left depicts the angles divided by the quantity of cameras in the unity game engine while the figure on the right shows that the quantity of projectors for use and the angle of view of the projection are identical. 

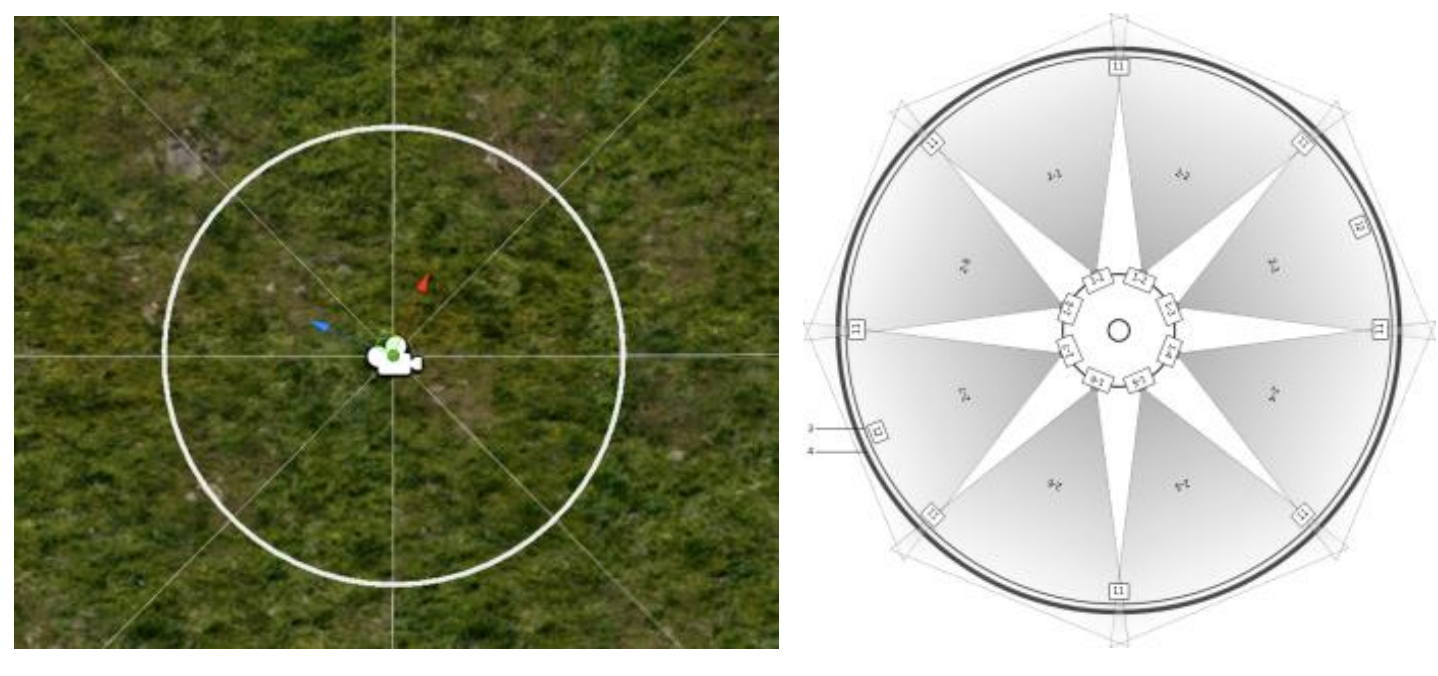

Figure 3. Game Engine / Projectors

Figure 4 illustrates a marked camera on the screen of the unity game engine. The marked camera allows visual image information that has been obtainted from the area of 45 degrees from $315^{\circ}$ to $360^{\circ}$ which is allocated to the camera - to be played on screen with the help of the projector.

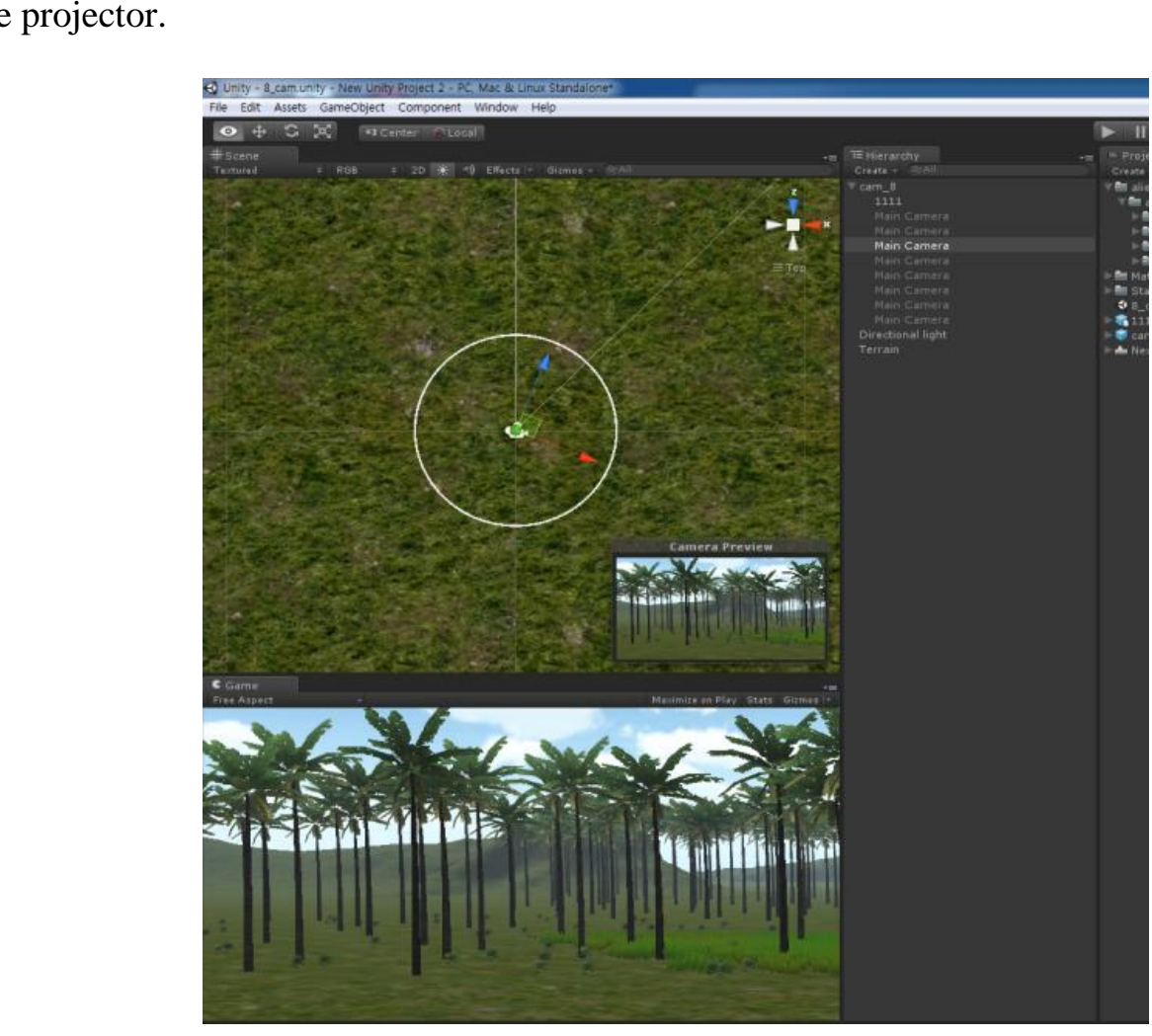

Figure 4. Camera Preview of Game Engine

When projecting the image information obtained from the camera, the correlation of the display dimension, overlapping ratios, angles of projection, etc. can be calculated as shown in Table 1. 
Table 1. The Calculation of Display Dimension

\begin{tabular}{|c|c|c|c|c|}
\hline \multirow[b]{2}{*}{ Area } & \multirow[b]{2}{*}{ Amount } & \multicolumn{3}{|c|}{ Diameter $6.35 \mathrm{~m}$} \\
\hline & & $\begin{array}{c}\text { Area \& } \\
\text { Angle }\end{array}$ & $\begin{array}{c}\text { Overlap } \\
20 \%\end{array}$ & $\begin{array}{c}\text { Real } \\
\text { Area \&Angle }\end{array}$ \\
\hline \multirow{2}{*}{ Image } & \multirow{2}{*}{$19.9 \mathrm{~m}$} & \multirow{2}{*}{$3.48 \mathrm{~m}$} & $\mathrm{~L} 0.5 \mathrm{~m}$ & \multirow{2}{*}{$2.5 \mathrm{~m}$} \\
\hline & & & $\mathrm{R} 0.5 \mathrm{~m}$ & \\
\hline \multirow{3}{*}{ Angle } & \multirow{3}{*}{360} & \multirow{2}{*}{$63^{\circ}$} & $+9^{\circ}$ & \multirow{3}{*}{$45^{\circ}$} \\
\hline & & & $+9^{\circ}$ & \\
\hline & & $306^{\circ}$ & $+9^{\circ}$ & \\
\hline
\end{tabular}

\subsection{Software Configuration}

When expressing a 360-degree panoramic picture through a unity game engine, the camera is realized in the form of a frustum. The following Figure 5 depicts the locations of eight cameras installed in the unity game screen.

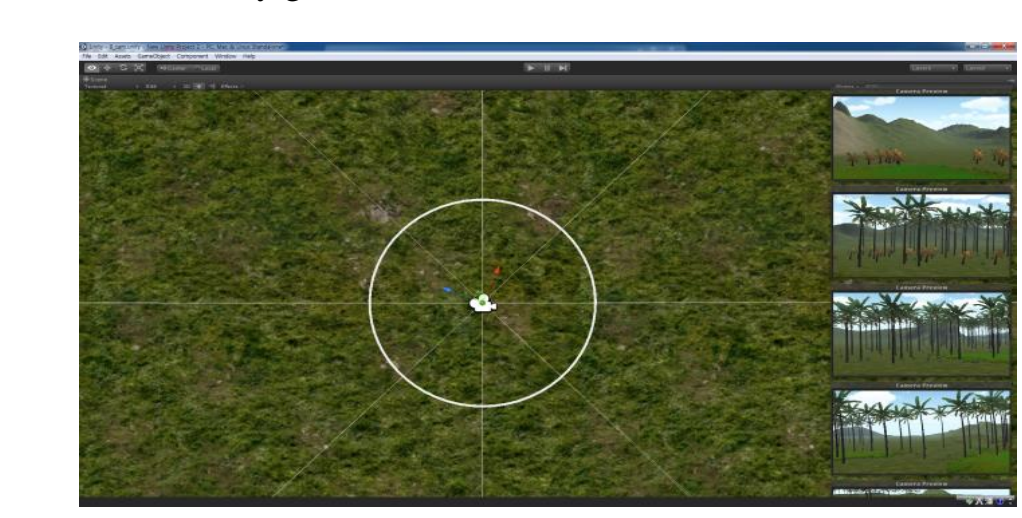

Figure 5. LCations of Camera Installed in the Game Engine

The image data obtained from the real-time hardware rendering, as shown in Figure 4, is projected on the 360-degree screen - by each determined area of the frustum - by the client PCs which are on the same network line [8]. A frustum in the unity game engine gets further as it shifts to the four corners based on the center of the screen. However, the four corners and the central part of the objects expressed on the screen are calculated to be in the same distance based on a point. Because of such attributes of the frustum, the objects which are actually located further than they seem are calculated to be in the same distance as the central part of the point.

For this reason, although it has to look smaller as it moves to the corners, distortion which makes it look bigger in the real-time rendered screen occurs. There seems to be no problem in the subject visually seen between the camera angles of $30^{\circ} \sim 60^{\circ}$, the distortion becomes more apparent as the angle gets larger than $60^{\circ}$, causing visual fatigue to worsen or interrupting the concentration of a viewer.

In this system, its realization is possible from an arc with a small angle of view of the camera to the perfect cylindrical 360 degree. Because of the attributes of the frustum in the 
game engine, the entire picture cannot be described only by a single camera but a panoramic picture should be segmented for display using several cameras. And because distortion occurs in the corner of each connected picture when the images obtained from the segmentation by several cameras are projected on the cylindrical screen to produce a single connected panoramic picture, proper levels of angles of view of the cameras should be applied to reduce this adverse phenomenon.

For general image display, for instance, when the angle of the camera is set at 45 degree, pictures divided by the angles of view of four cameras are produced in the 180-degree semicircular form while pictures by the angles of view of eight cameras are made in the 360degree panoramic picture. Although in this study, the shape of $6.35 \mathrm{~m}$ in diameter is the basis, when the size of the circumference that forms this system according to the display environment increases, the angle of view of the camera by the ratio of length and width should be adjusted and the quantity of projectors, client PCs, etc. will increase if threedimensional realization is necessary. Thus, to establish a networkenvironment needed for the synchronization of correct images, equipment with high-performance and high level of specifications and also high quality are necessary.

Figure 6 is a diagram of the Multi Display System (MDS) used in this system and it explains the concept that each camera in the unity game engine outputs the information, which has been produced in the area of FOV (Field of view) $45^{\circ}$ and size $768 \times 768$ pixels, in the grid-set screen zone after rendering it in each client PC.

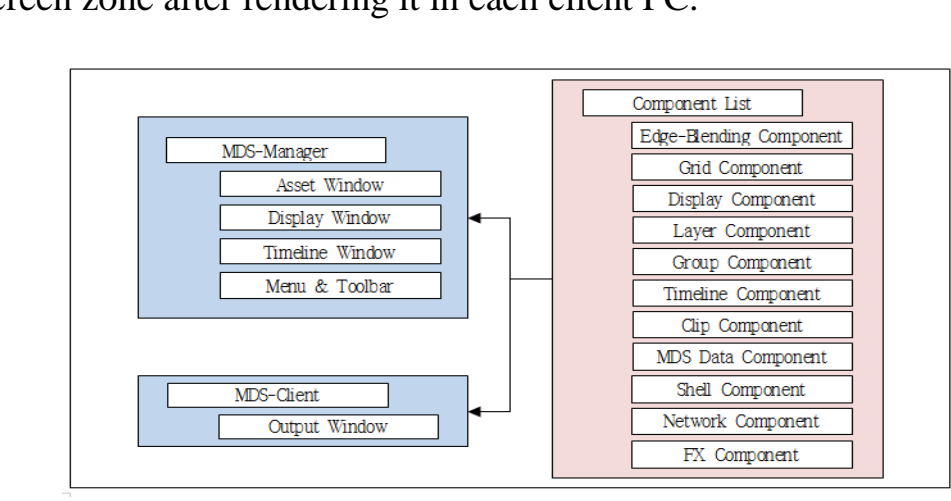

Higure 6. Multi Display System (MDS)

Executing the acyiewer of the Multi Display System (MDS), access should be made by tagging the following detail in the firstdata.xml file and through the IP and port of the administrat r who intends to access: <network server="192.168.206.254" port="4711" client id="client001" password="client001"/>. And according to the order of accessing to the acAPP Test Server, the index value is assigned beginning with no. 0 . In other words, if the access has made by the order of btp-c01, btp-c02, btp-c03, btp-c04 and so on, index is assigned as each $0,1,2,3$, and so on. And commands with the viewer computer accessed through the set client commands in the acAPF_Test Server can be confined. Also, when a command of set client 0 is entered, control command for the btp-c01 computer can be delivered. When set client -1 is commanded, control command is simultaneously delivered to all computers that have been accessed.

As shown in the 5-1 3 of Figure 7, the picture of projecting the camera information in different locations installed in the unity game engine on the cylindrical screen should be output on the screen without variation through each projector in the 4-1 8 of Figure 8 and at certain locations with certain time. 

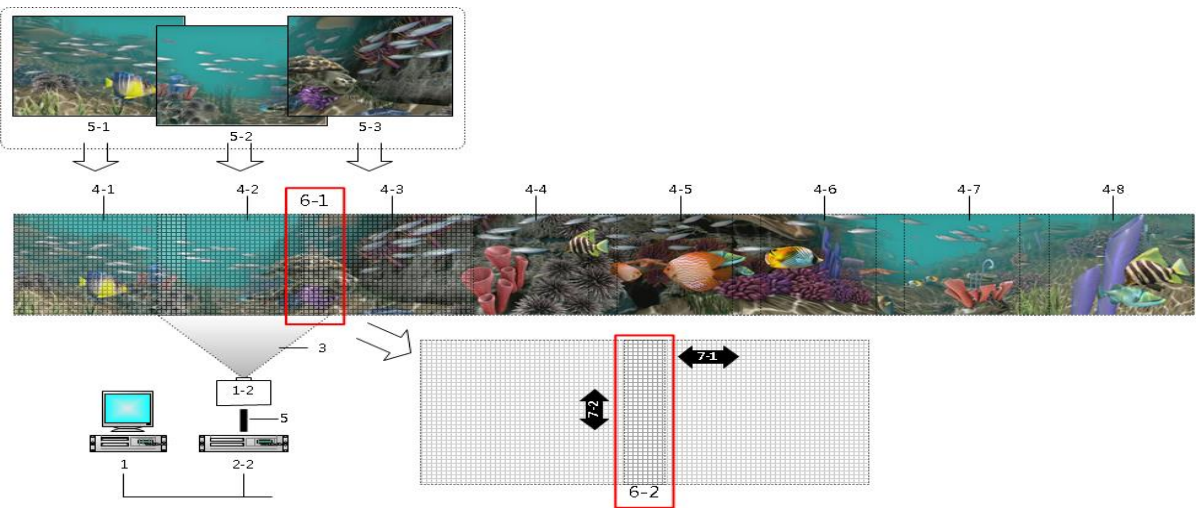

Figure 7. Edge Blending Technology

At this moment, because of the attributes of the projector device and scteen, differences occur in the quantity of light and focus as they shift from the center of the screen to each corner and when a single picture is output by connecting a panoramig picture through several projectors, the edges of the vertical border lines become apparentin the area in which the frames of each image that the projector projects are overlapped. To gain the natural overlapping effect of the frame of images projected between projectors as shown in the 6-1 of Figure 7, an edge blending technologycshopld be applied in the connected area of each overlapped frame. At the same time, it solves theshorizontal axis and the longitudinal axis of the grid by the technical processing as shown in the 6,2 of Figure 7.

In this study, as 6-2 of Figure 8 the edge blending value in the linear method has been applied to the overlapped images as shown 8 of Figure 8 , the level of gamma within $20 \%$ of the overlapping range has been adjusted and gradation and the transparency by level have been applied to the brightness of particular area which utilizes such technique as adjusting the transparency of the gradation and the part where images are overlapped as shown 6-3 of Figure 8 .

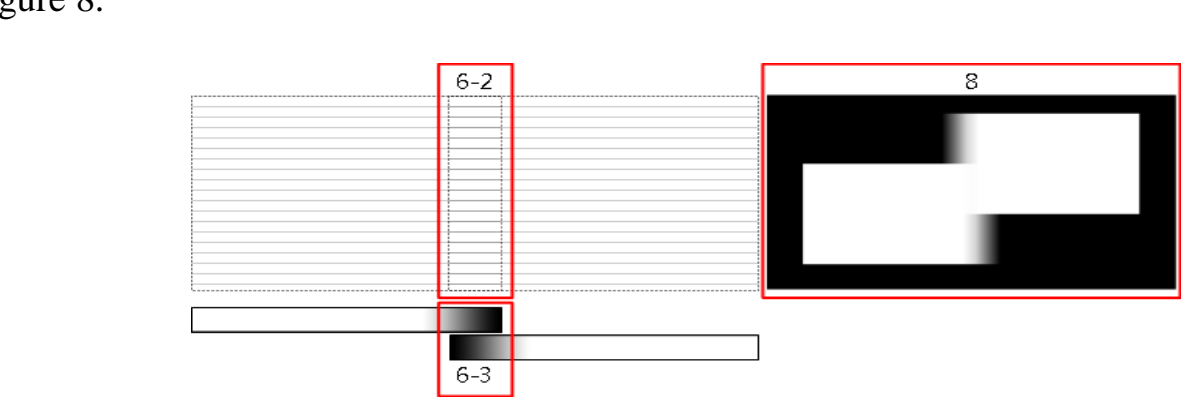

\section{Figure 8. The Edge Blending Value}

The shell commands given from the window of acViewer of the Multi Display System (MDS) include:

Table 2. The Shell Commands

\begin{tabular}{|l|l|}
\hline Setclient $0[$ Enter] & Sets to control the btp-c01 c08 viewer. \\
\hline Setclient -1 [Enter] & Controls the entire viewer. \\
\hline
\end{tabular}




\begin{tabular}{|l|l|}
\hline Gridedge 0 [Enter] & Cancels the edge blending. \\
\hline Gridedge 2 [Enter] & Sets the linear edge blending on both sides. \\
\hline Wireframe & Toggles the wire frame in the rendering option. \\
\hline Autoapply & $\begin{array}{l}\text { Toggles whether the display apply should be automatically } \\
\text { executed. }\end{array}$ \\
\hline
\end{tabular}

The test process of the linear edge blending method, as shown in Figure 9, has significant differences between the bright screen Figure 9 (a) and dark screens Figure 9 (b), (c); it has been verified that natural images can be obtained in the entirely dark screen. As described earlier, in the bright screen as well, the overlapped area of the vertical border lines can be adjust in a more natural way by adjusting the level of gamma, the level of transparency of the gradation, etc.

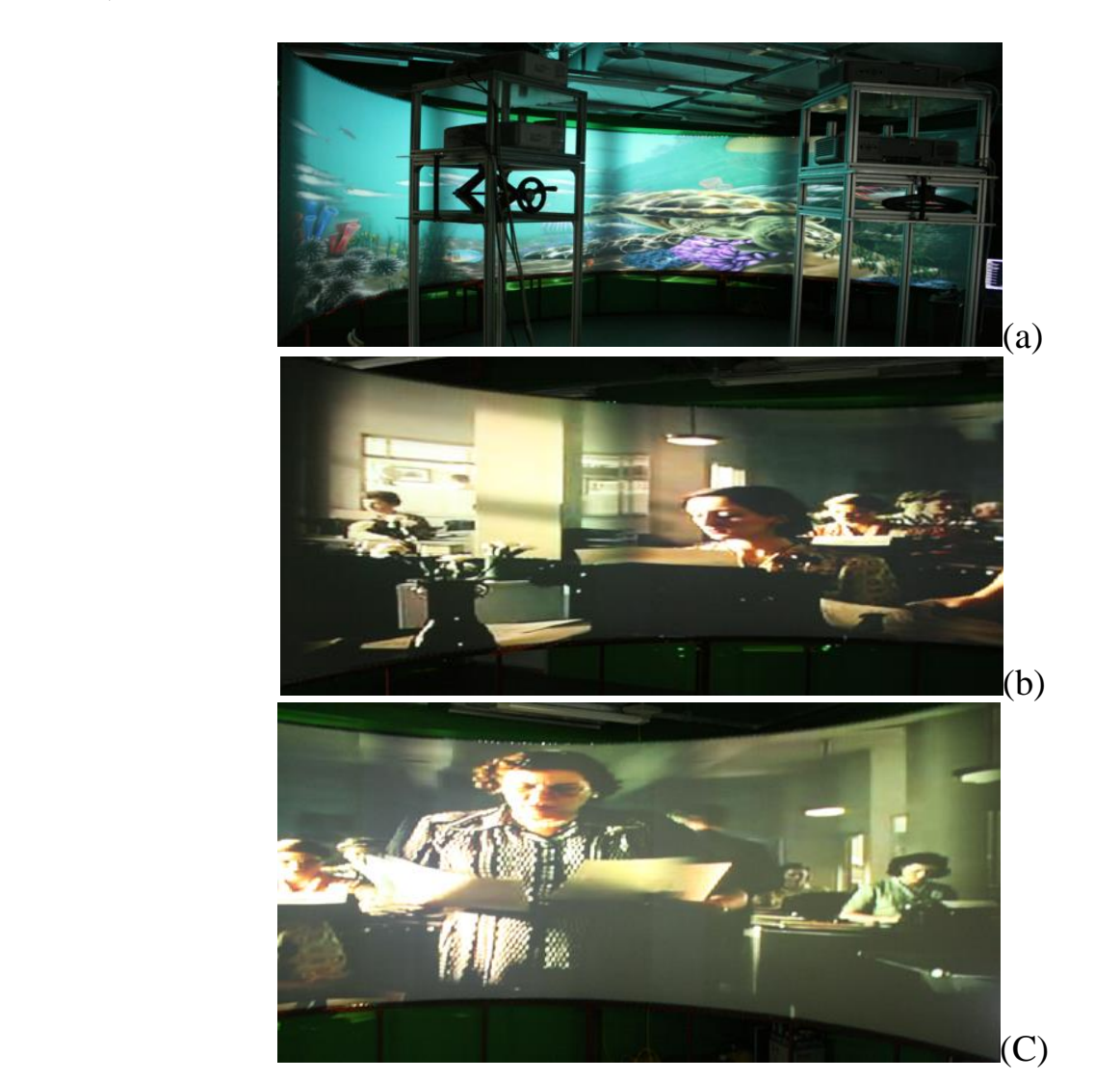

Figure 9. Linear Edge Blending Test (a), (b), (c)

When a picture is projected through the straightness of the light by using a cylindrical curved screen but not a flat screen, distortion occurs on the screen image projected through the projector. To reduce the distortion that takes place due to the difference in the distances of the image of the frame projected in a way described above reaching to the center of the curved screen and of the corners, the grid system for handling the wrapping of right images in the software where a frame is output should be realized and the distortion of the image be remapped right in the curved surface of the screen. In this study, the grid is basically adjusted 
from each viewer in five equal parts horizontally (six x-direction index) and in one part vertically (two y-direction index). The quantify of grid can be increased if necessary, as shown in Figure 10; however, as it increases, the moving width of the grid becomes narrow which consequently makes it more difficult to match the continuity of the images that are generated in the horizontal section.

The shell commands of grid system given from the window of acViewer of the Multi Display System (MDS) include:

Table 3. The Shell Commands of Grid System

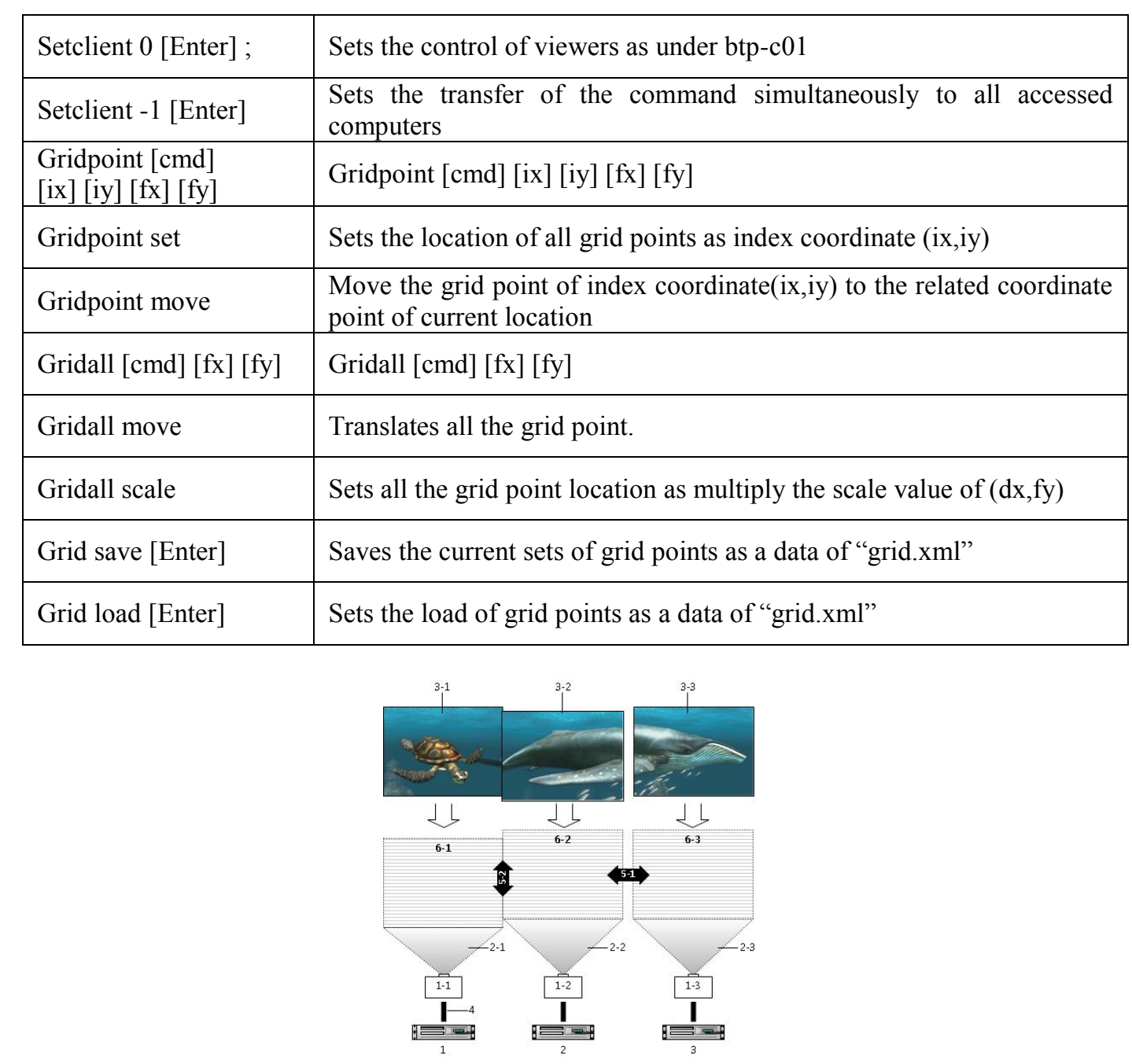

Figure 10. The Example of Grid System

\subsection{Hardware Configuration}

The establishment of a cylindrical multi display system normally requires such hardware module as a cylindrical screen structure, picture projection equipment, network and synchronization, etc. Figure 11. 


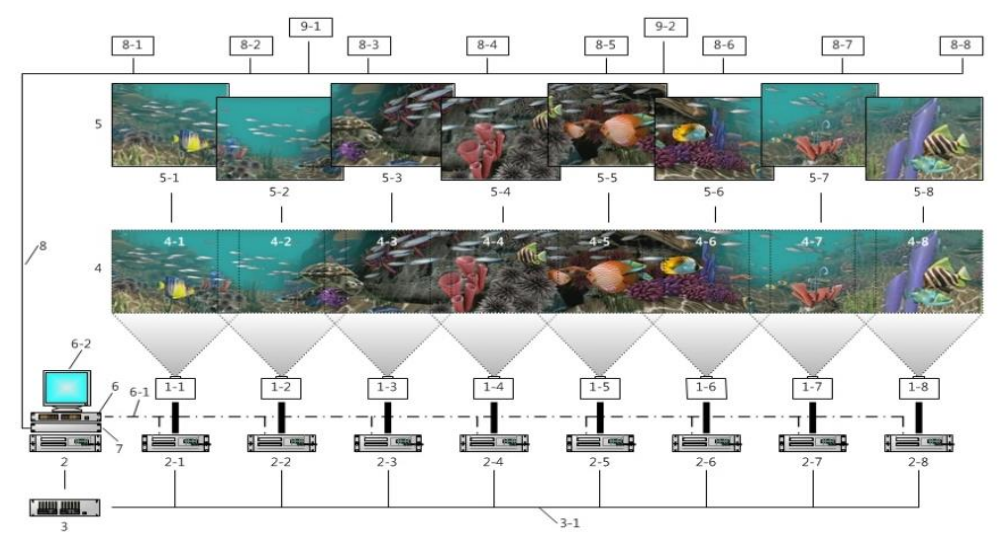

Figure 11. Hardware Diagram .

The projectors should distribute a more than one preture that has to be connected to multiple PCs real-time. When realizing a three-d mensional picture, the same quantity of projectors and client PCs - which is eight respectively - shot ld be additionally installed or 2D or 3D images can be realized by loading two graphic cards in each client PC that has been initially designed [9].

Thus, the location of the projector can be confirmed depending on the size and distance of the screen when projecting images from the projector For an ordinary projector, since its projection distance is long, a proper-syed diameter should be determined beforehand in order to overcome the projection distance when installing this system. In order to overcome the limits of the cylindrical structure which is $6.35 \mathrm{~m}$ in diameter, a single vision lens was used and the projector was placedin the uppen side of the screen in this project, as illustrated in Figure 12, to prevent the viewers frombloeking the projected pictures in such narrow space or to secure more proximity in order to overcome the limits of the cylindrical structure which is $6.35 \mathrm{~m}$ in diameter.

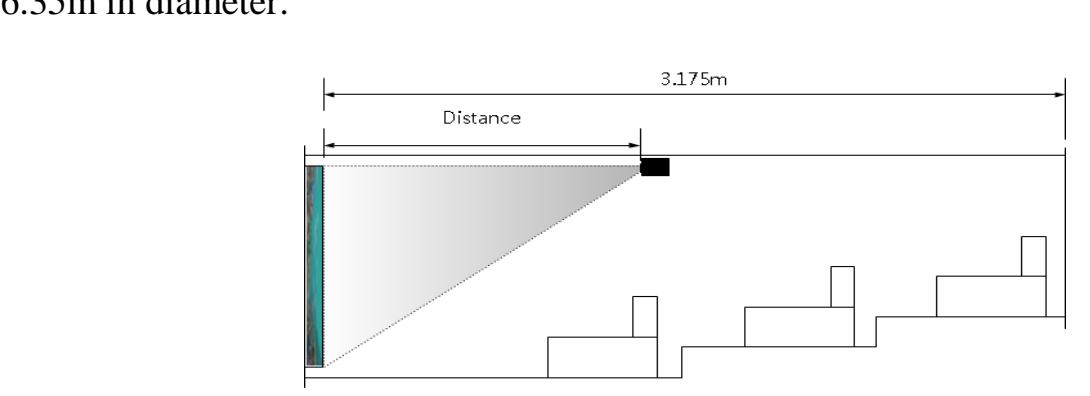

Figure 12. Game Engine and Projector

To sustain the quality of the final images, it is very important to select a projector and specifications that are suitable for the environment. Projectors can largely be divided into LCD and DLP; an LCD has an excellent brightness and splendid colors compared to the same ANSI lumen rating of a DLP while a DLP has a great contrast range. In this study, a 3000 ANSI lumen DLP was used to realize the cylindrical vision.

\subsection{Network Configuration}

The pictures to be connected by multiple projectors should be synchronized and output like a piece of figure. At the same time, since there are limits of quantity of data that a single 
computer can control, a number of computers should be accessed to the network and control the multiple projectors simultaneously through the network synchronization communication.

The image projection equipment is composed of eight projectors, eight client PCs, and a control server PC and it projects images and movies when it displays $2 \mathrm{D}$ images. To realize 3D pictures, additional eight projectors and eight client PCs should be installed.

It outputs each source picture set up on FOV (field of view) $45^{\circ}$ of camera view of the game engine as images by hardware rendering through each client PC at the size of $768 \times 768$ pixels. Each client PC is a projector physically connected; it simultaneously outputs images or pictures of the grid setup area within the 45 degree of the 360 degree cylindrical displayed view screen partitioned by eight areas and the signal processing which is sent to each client $\mathrm{PC}$ from the server is $30 \mathrm{frame} / \mathrm{sec}$ and network time synchronization should be done by force every $33 \mathrm{~ms}$ (millisecond) [10].

The commands of the management of client PC given from the window of acViewer of the Multi Display System (MDS) include:

Table 4. The Commands of the Management of Client PC

\begin{tabular}{|c|c|}
\hline Video $[\mathrm{cmd}][$ index $]$ & Video [cmd] [index] \\
\hline Video file [index] [file] & Registers an intializes the video file in the index from 0 to 8 \\
\hline Video play [index] [dtime] & Plays, the registered video file from cue in the index \\
\hline Video pause [index] & deo in the index \\
\hline Video stop [index] & the video playing and sets timeline as zero point $(0)$ \\
\hline Video del & Defetes the registered video file in the index \\
\hline View $[\mathrm{cm}$ & Oommands for video screen location \\
\hline View pos0 & Changes the screen location of registered video of 'start' \\
\hline View pos1 [index] & Changes the screen location of registered video of 'End' \\
\hline View uv0 [index] [uo] [v0] & Changes the texture location of registered video to 'Start'. \\
\hline View uv [index] [u1] [v1] & Changes the texture location of registered video to 'End' \\
\hline Display [cmd] & Display [cmd] \\
\hline Display init & $\begin{array}{l}\text { Initializes the basic information of display from document. } \\
\text { (If components would be automatically set after initialization.) }\end{array}$ \\
\hline Display add & $\begin{array}{l}\text { Adds the view information of video in the display and sets the } \\
\text { grid. }\end{array}$ \\
\hline Display set & $\begin{array}{l}\text { Sets the grid information of display into the information of render } \\
\text { loop and executes the rendering }\end{array}$ \\
\hline display apply (add + set) & $\begin{array}{l}\text { After sets the grid of video view information, sets the information } \\
\text { in the render loop }\end{array}$ \\
\hline
\end{tabular}




\subsection{LOD Function}

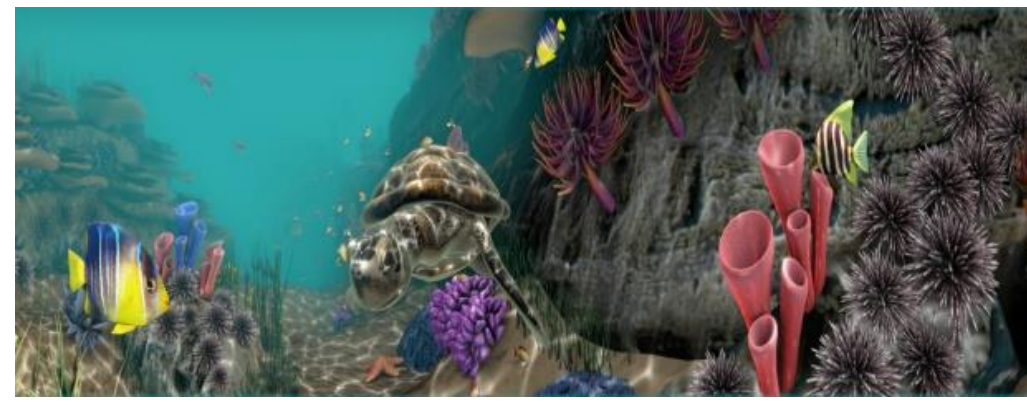

Figure 13. The Example of LOD Apply

For the design resources such as characters and backgrounds produded by the 3D CG technique, the LOD (Level of Detail) is applied to overeome the limits of the game engine and sustain their more effective visual high quality figure 13. In this study, a screen zone was designated largely in three hierarchical structures - the front, central and back sides - by the distance base reflected through the eight cameras used to realize 360-degree panoramic images. And LOD was designated to apply the quantity of polygon such as low, mid, and high for relative data structure and, for the eontents, a basic 3D modeling resource was produced according to the standard specified in Table 52

\section{Table 5. LOD Rolygon}

\begin{tabular}{|l|c|}
\hline Subdivision & Number of Polygon \\
\hline High poly model & $2,000 \sim 10,000$ \\
\hline Low poly model & $15,000 \sim 20,000$ \\
\hline
\end{tabular}

Table 6 and Table 7 describe the concepts of the application of LOD Type according to the focus of the camera.

Table 6. LOD Apply with Focus Locking

\begin{tabular}{|l|l|l|l|}
\hline \multirow{2}{*}{ LOD } & Front sides & Central sides & Back sides \\
\cline { 2 - 4 } & High polygon & Mid polygon & Low polygon \\
\hline
\end{tabular}

Table 7. LOD Apply with Focus Moving

\begin{tabular}{|c|c|c|c|c|}
\hline L & M & Front sides & M & L \\
\hline L & M & Central sides & M & L \\
\hline L & M & Back sides & M & L \\
\hline
\end{tabular}

To express the details of each object, various texture maps such as diffuse map, normal map, occlusion map, and light map have been used and the occlusion map or light map tends to be used by blending them with the diffuse map. And to express the three-dimensional 
structure, the three-dimensional space whose lighting and shading are processed and expressed on computer is eventually output by the two-dimensional display device. The following is the process in which the three-dimensional space and objects are transformed into the two-dimensional display:

3D left Table (x, y, z) --> View conversion (view matrix) --> Projection conversion (Perspective matrix / Orthogonal matrix) --> 2D Display left Table (x, y) conversion.

\section{Performance and Installation}

\subsection{Rendering Performance Evaluation}

The real-time hardware rendering is a technology that has been realized to express threedimensional computer game images. In a game, the objects are expressed on the screen realtime by its interaction with the user. To make this happen, the real-time/rendering process is needed to express these objects in the game which are being praced individully in the threedimensional space and a matrix - a mathematical technique-1s used for them to be expressed in visual images. A unity game engine is used as too 1 to realize surch real-time rendering.

The rendering formation process includes the image-based sotware rendering Figure 14 which utilizes the graphic application (Maya, 3dX MAX, Light wave, etc.,) such as Ray caster and Ray tracer and the real-time hardware rendering Figure 15 which uses a game engine among those rendering methods adopted for the production of contents. Each rendering method carries out the rendering in completely different ways in the procedures for the production of pipe lines and the formation method of data for rendering or the equipment and software used can also be modified of their partial procedures in a more special and dense way depending on the size of the images or scenes to be displayed. The image-based software rendering which uses 3D apflications such as Maya and 3ds Max perform additional render processing to revise ${ }^{\bullet}$ inages that freguently occurs while being developed causing inconvenience to work ony

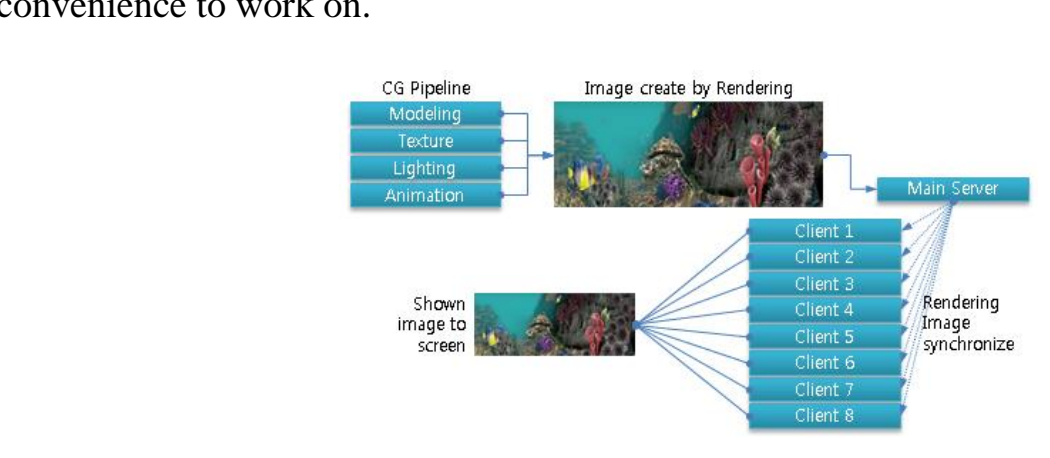

Figure 14. Software typed Rendering Pipeline

The significant purpose of rendering is identical because each graphic asset produced in a $3 \mathrm{D}$ application is combined in the game engine for the last time to display pictures; however, compared to the existing software-based rendering procedures, modification is easier and system establishment for additional processes is not necessary which makes the process a lot simpler Figure 15. 


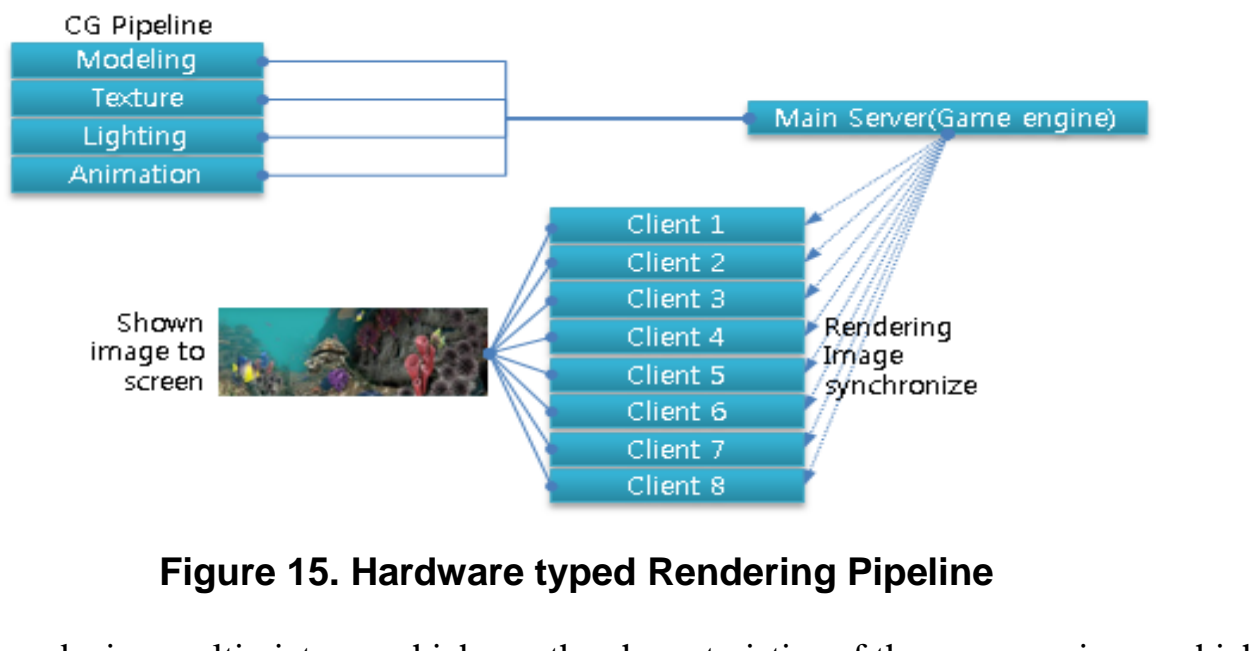

When producing multi pictures which use the characteristies of the game engine, - which is also the greatest attribute in this study, - the camere divided by angle in the formation of 360degree scene, in other words, when rendering the scenes seen fn the frustum, the pipe line of each method has been simplified and efficiency of the run-time of rendering has been made. The run-time has declined by at least ${ }_{0} 1 / 20$ through which rapid processing results are expected [11].

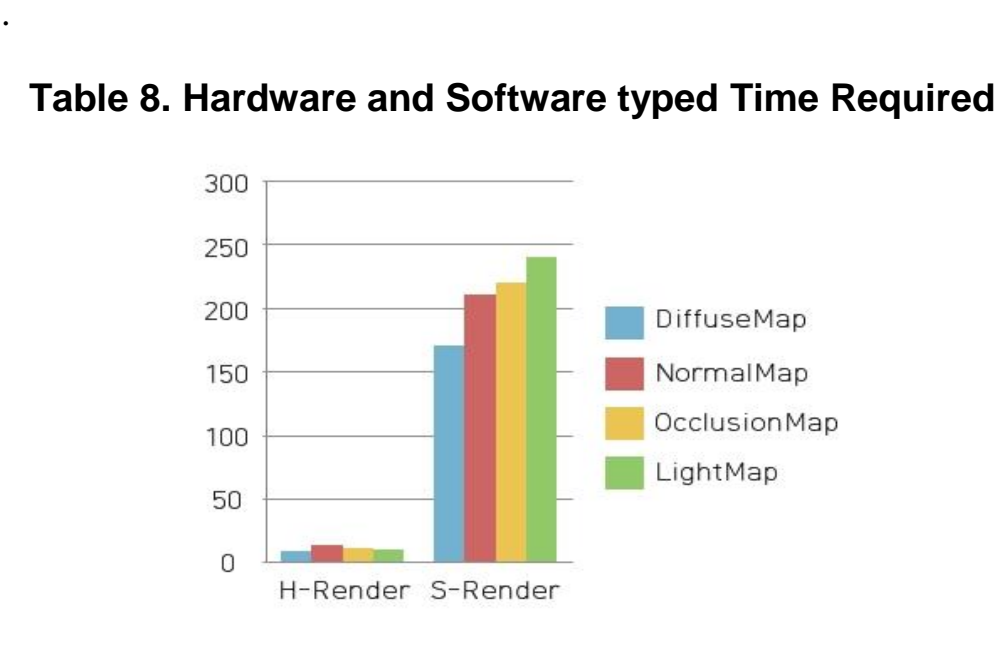

When rendering basic pictures based on the order of render layer used to process effects such as reflections, refractions, and shadows which are the functions through which shapes are reflected real-time, the refraction of light occurs when projected, or afterimages are constant $y$ output in the table side of other objects in the environment in which the run-time of rendering varies according to the use and attributes of diffuse, normal, occlusion, and light maps, and rendering is needed, a great comparative value appears in the image generation.

For more efficient adjustment of the rendering time and optimization of the picture, when forming the initial texture map, occlusion or light map can be extracted in advance and blended to the diffuse map to reduce the quantity of render layer during the rendering process while, in the system, since only two textures including diffuse map and normal map are rendered, such effect as faster run0time can be obtained on Table 9.

Table 9. Hardware and Software typed Time Required 


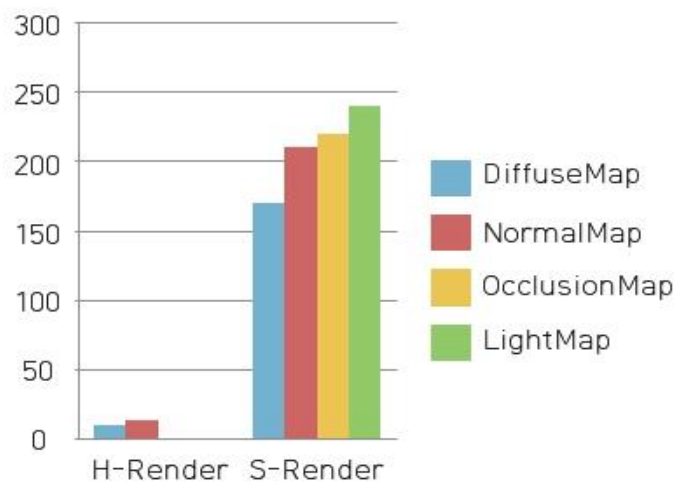

In case of the normal map, rendering workload and time reduction occur by selectively using the objects scattered in the game scenes or by blending the concep of unevenness to the diffuse map by the image description method when forming a texture map on Table 10.

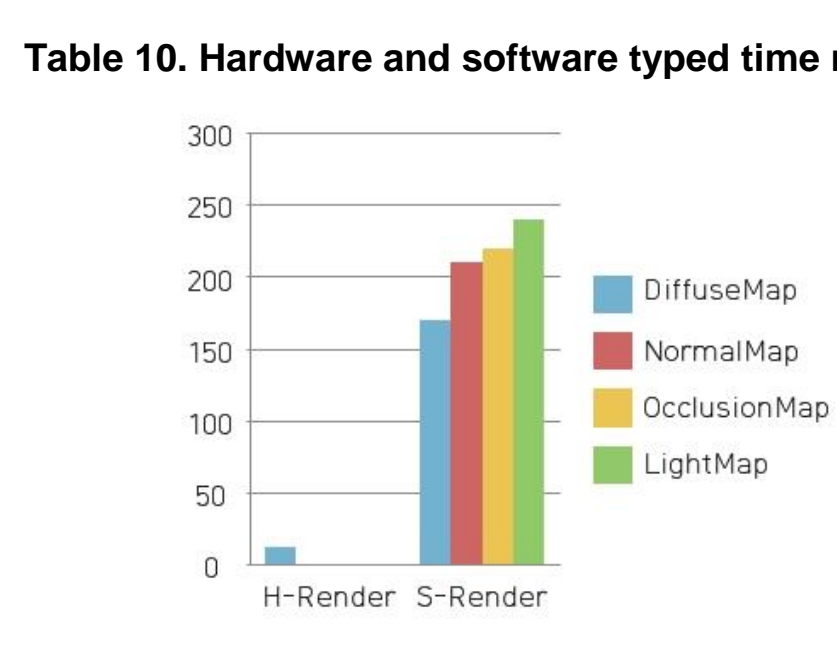

\subsection{Installation of a Cylindrical Screen Structure}

The important point is that, for a structure, if such wall materials as plywood are used, paint should be applied evenly to the entire area and if installed with a tent,

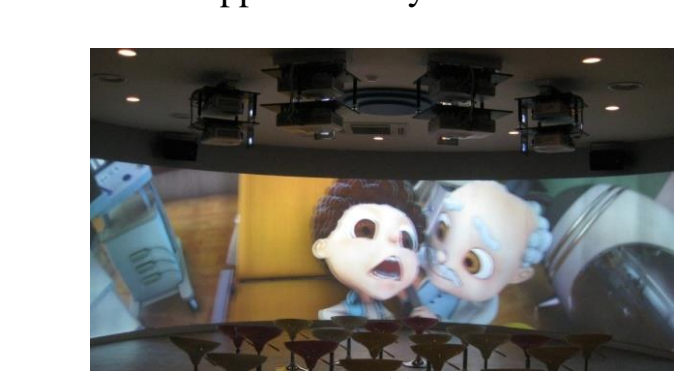

(a)

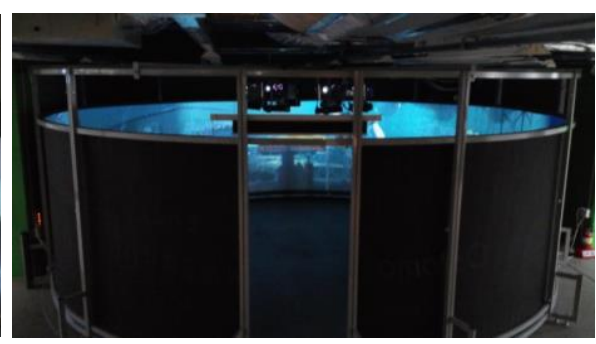

(b)

Figure 16. (a) Half Cylindrical 3D Multi Display System, (b) Cylindrical 3D Multi Display System

For the correct 3D image projection, however, specially processed paint for silver screen use should be applied to the cylindrical structure or a special fabric material silver screen 
needs to be installed. The important point is that the fabric should be exactly cut out and be firmly fixed tightly and evenly to the four sides on the stand. The height can be adjusted by the one who designs - from bottom to top - within the limit that the structure of the building allows. In this case, the intensity of illumination of the projector (ANSI, LUMEN) and the performance of the lens (the focal length) must be checked to determine an optimized model.

We have built several prototypes. The first 3D half cylindrical multi display system is 2.62 meter $\times 9.97$ meter (height $\times$ circumference) which includes 2 each $\times 4$ set Epson EB-G5800 projectors with ATI Radeon 6900 graphics board on Figure 16 (a). The length of a cylindrical silver screen is $19.939 \mathrm{~m}$ which is derived from the calculation of the circumference of $6.35 \mathrm{~m}$ in diameter. It has 2 each $\times 8$ set Optoma projectors with fish eye lens and ATI R'adeon-R9 290 graphics board attached on top ceiling to a circular fixture, as illustrated in Figure 16 (b).

The material of the silver screen has elasticity because it has gone through the coating process with the use of a thin cloth applied with special silver paint which can makes it possible for three-dimensions to be realized. To fix the sereen onto the fixture, a thin plastic film is added to the edge of the screen fabric and about $5 \mathrm{~cm}$ holes are made Figure 17 (a), and adjusted around $5 \mathrm{~cm}$ apart from one another and comnected with the use of an elastic string depending on the installed fixture and distance on Figure 17 (b)

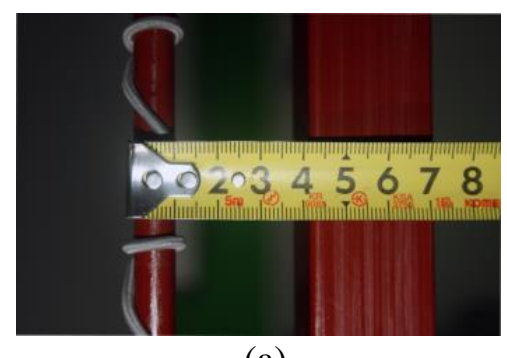

- (a)

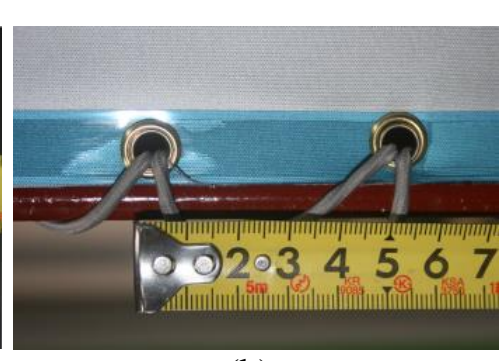

(b)

Figure 17. (a) The Supporting Frame and Screen Supporting Frame, (b) The Ground-connection of the Screen

This method of production reduces the expansion and worn-out of the end of the screen and the edges in whieh wires are seen are neatly patched by the molding process. The connection should be made from the central part so that the pulling strength with the help of tension of the screen can be constantly functioning as shown in Figure 18.
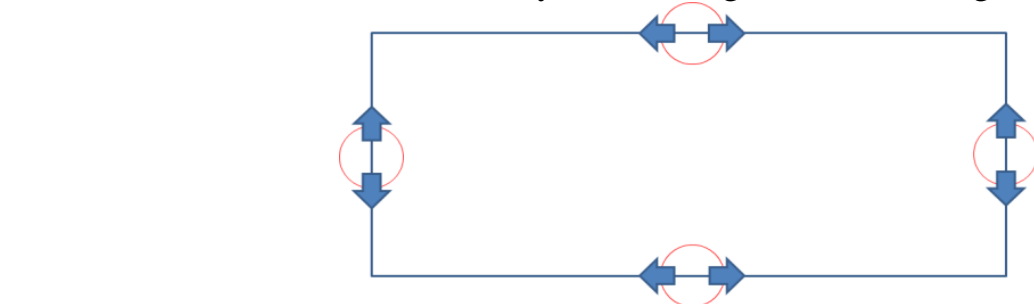

\section{Figure 18. The Working Direction of the Wire with the Continuance of the Tensile Strength of the Screen Taken into Consideration}

The expandability of the installation system which has been understood in the study can realize the height and width according to the diameter as much as the projection distance of a projector permits. 


\section{Conclusion}

\subsection{Differentiation}

This study has been conducted on the cylindrical multi display system which can maximize the experience of viewers in theme parks and experience theaters by extending the environment in which a viewer can concentrate his or her visual experience. For this purpose, this study has offered efficient results in terms of time and cost both by enabling the re-use of CG resources, easy updating and real-time image processing by the hardware rendering method that utilizes a game engine. One of the advantages of this system, in particular, is that it can also realize a three-dimensional display.

\subsection{Associated Fields for Further Development}

We have implemented the first work to multi display on cylihdrically extended screen with using multi projectors. The hardware rendering method to use the game engine has great effects for improvement in terms of time and economic fáctors by processing images realtime. In the aspect of localization of technology that has been oversea dependent, the enhancement of competitiveness including the reduction on expenses and efficient development can be further applied in diyerse-fields ${ }^{\circ}$ such as theme parks, exhibitions, education, etc., through picture production and expression know-how optimized for family entertainment centers and theme park-typed entertainment industry. This study will be of industrial significance to attractions wich will have a tremendous popularity when the circlevision system is combined with three-dimensional image contents and interactive game technology. [12] It will be led to the development of contents that specialize in interactions according to the characteristics of the game engine which will be utilized to create new spaces.

\section{Acknowledgements}

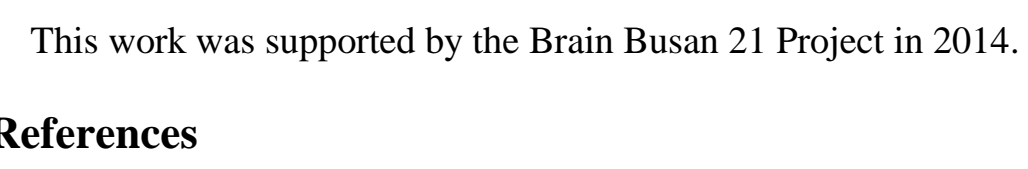

[1] H. I. Kwon, H. J. Rhee, and J. W. Park, "Exploring the Immersion Degree Difference Between 3D and 2D: Focus on Action-A dventure Game", Journal of the Korea Contents Association, vol. 11, no. 1, (2011).

[2] A. Majumder and S. M. Brown, "Practical Multi-Projector Display Design", A K PETERS, Ltd, Wellesley (2007).

[3] D. Vogel and K. Balakrishnan, "Interactive Public Ambient Displays: Transitioning from Implicit to Explicit Public to Personal", Interaction with Multiple Users, Proceedings of UIST, (2004) October 24-27, Santa Fe, NM. USA.

[4] CS Park, Kj, Lee, Nh, Kim, Sh lee, Ky Seo and Ks, Park, "Design and Development of Multiple Input Device and Multiscale Interaction for GOCI Observation Satellite imagery on the Tiled Display”, J. Korea Inst. Inf. Commun. Eng. vol. 18, no. 3, (2014).

[5] G. Humphreys, M. Houston, R. Ng, R. Frank, S. Ahern, P. D. Kirchne and J. T. Klosowski, "Chromium : A Stream Processing Framework for Interactive Rendering on Clusters", Proceedings of SIGGRAPH Conference (2002) July 21-26; San Antonio, TX, USA.

[6] B. Jeong, L. Renambot, R. Jagodic, R. Singh, J. Aguilera, A. Johonson and J. Leigh, "High-Performance Dynamic Graphics Streaming for Scalable Adaptive Graphics Environment", Proceedings of High Performance Computing, Networking, Storage and Analysis (2006).

[7] H. S. Pak, H. J. Jeon and E. J. Chae, "The Implementation of Large-Scale Circle-Vision System for the Environment of Theme Park-Typed 3D Contents", Advanced Science and technology Letters, vol. 47 (Culture and Contents Technology) (2014) April 15-18; Jeju Island, Korea.

[8] H. N. Lee and W. H. A. Lee, "Frustum-Based Real-Time Ocean Rendering with GPU", Journal of the Korea Game Society, no. 10, (2007). 
[9] A. Majumder and M. S. Brown, "Practical Multi-Projector Display Design", A.K.Peters, Ltd. (2007).

[10] R. Raskar, J. V. Baar, P. Beardsley, T. Willwacher, S. Rao and C. Forlines, "iLamps: Geometrically Aware and Self-Configuring Projectors", Proceedings of ACM SIGGRAPH Conference (2003) July 27-31, San Diego, CA, USA.

[11] P, Harish and P. J. Narayanan, "Designing Perspectively-Correct Multiplanar Displays Center for Visual Information Technology", IEEE Transactions on Visualization and Computer Graphics, vol. 19, no. 3, (2013).

[12] C. O. Yun, T. S. Yun and D. H. Lee, "Spatial Interaction System for Providing Interactive Zone in LargeScale Display Environment”, Proceedings of HCI International (2009) July 19-24; San Diego, CA, USA.

\section{Authors}

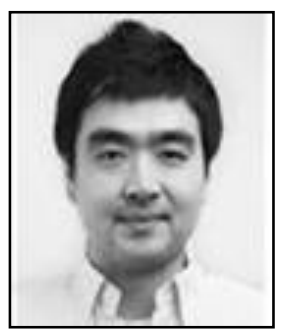

Hyungjin Jeon, BFA and MFA of Photography from Chung Ång Univ. Seoul Korea $(2009,2012)$

Ph.D. Course at Division of Visual Contents, Graduate/Sckool, Dongseo Univ. Busan, Korea (2013 )

Research interests: Digital Media, Visual Contents

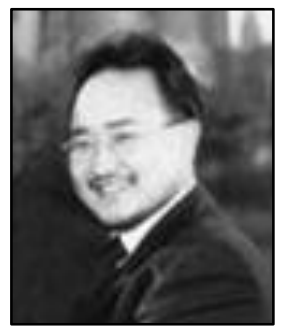

Eeljin Chae, BFA of Photography from Chung-Ang Univ. Seoul Korea (1998)

MFA of Motion picture from Acadeny of Arts Univ. USA (2001)

Producer for TUBE PICTURES, FX Digital, (2001 2004)

Associate Professor at the Division of Digital Contents, Dongseo Univ.

Busan, Korea (2004 )

Researchinterests: Digital Media, VFX, Contents Business

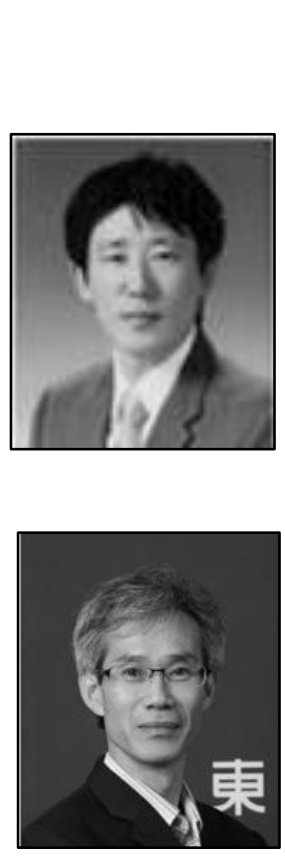

Hongsik Dak, MFA of Computer arts from Academy of Arts Univ. USA (2000)

Associate Professor at the Division of Digital Contents, Dongseo Univ.

Busan, Korea $(2010 \sim)$

Research interests: Interactive Contents, Game Design

Taesoo Yoon, Ph.D. of Computer Engineering from Kyungpook National Univ. Daegu, Korea (2001)

Researcher for DAEWOO Electronics R and D Center (1993 1998)

Full Professor at the Division of Digital Contents, Dongseo Univ. Busan, Korea $(2001 \sim)$

Research interests: Game Development, A.I. 